\title{
Decoupling Analysis of Input Parameters of Cubic Mechanism
}

\author{
Jian-Guo LUO' ${ }^{1}$, Jian-You HAN ${ }^{2}$ \\ ${ }^{1}$ North China Institute of Science and Technology, Mechanical \& Electrical Department, Beijing, \\ 101601 \\ ${ }^{2}$ Beijing University of Science \& Technology, School of Mechanical Engineering, Beijing, 100083 \\ luojg_1598@163.com
}

Keywords: Cubic Mechanism, Input Parameter, Configuration, Decouple.

\begin{abstract}
A new cubic mechanism with 6 degree of freedom based on the $\mathrm{S}(\mathrm{P})[\mathrm{T}]$ output base and $P$ input base presented, upper and lower chains composed of up and down, back and fore, left and right linear drivers, two chains connected with the hooke joint and ball joint respectively, a passive $\mathrm{P}$ joint connect ball joint and hooke joint as the output spindle actuator. Based on the conclusion of kinematics analysis, response of the six straight line input parameters to spatial position and posture of output shaft analyzed respectively, which verified by examples, results obtained that independent control function of the upper moving platform mechanism to the pose of output shaft, still independent control function of the lower moving platform mechanism to the position of output spindle, each input parameter corresponding to the control of position components and pose components, which has played a very good role in decoupling.
\end{abstract}

\section{Introduction}

Serial mechanism and parallel mechanism have its advantages and disadvantages respectively, its advantages and disadvantages have complementary relationship, also has the dual relation in the structure and performance characteristics. From the comprehensive effects of workspace and $\mathrm{CNC}$ axis ratio and the ratio of performance to price and so on, pure parallel mechanism is not necessarily the best, but the hybrid mechanism with serial and parallel structure has more use value [1]. Serial-parallel mechanism has the advantages of traditional serial mechanism and parallel mechanism together, it is a kind of promising [2] CNC machine tool. In 2001, Zhao Wei[3] put forward a new serial-parallel micromanipulator experimental system for micro-operation, experiment results show the effectiveness of its composition and control and calibration method to complete high precision micro operation task. Xu Liju[4] proposed a hybrid virtual axis machine tool for multi coordinates NC machining with 4-DOF spatial parallel mechanism as main feed mechanism and a two-way moving worktable. Liu Haitao[5] research a 5-DOF hybrid reconfigurable robot with a 2-DOF spherical parallel mechanism and a serial kinematic chain of two rotation one translation, which connected with a sliding pair. In 2002, robots and mechanical design lab of California University of USA show the world a new 3-DOF universal Cartesian coordinates serial-parallel manipulator UCR[6]. This paper presents a new type of hybrid cubic mechanism[7] based on $\mathrm{S}(\mathrm{P})[\mathrm{T}]$ output base $((\mathrm{P})$ means passive telescopic joint, namely the auxiliary joint, $[\mathrm{T}]$ means the output joint) and $\mathrm{P}$ input base, as shown in Fig.1, 6-DOF motion realized through the spatial three dimension linear driving ${ }^{[8]}$. Analysis on the input parameters of position and pose of output shaft carried out here, decoupling attitude of each input parameter to the position and pose of output shaft obtained then, simulation results provide effective judging base as well.

PPP-S(P)[T]-PPP hybrid cubic mechanism as shown in Fig. 1, the components assembled on a cubic framework consists of upper and lower branches. Hooke joint of lower branch connected with a slider can move front and back, the guideway of this slider fixed on a slider can move left and right, the guideway of this slider fixed on a slider can move up and down. Ball joint of upper branch connected with a slider can move front and back, the guideway of this slider fixed on a slider can move up and down, the guideway of this slider fixed on a slider can move left and right. Ball joint 
connected to the hooke joint through a passive variable length translation pair, which construct the output shaft. Cartesian coordinate system built based on the mechanism, the name of each part as shown in Fig. 2, in order to increase the rigidity and stability of the structure, auxiliary support rail described in dotted line shown in Fig. 1, both ends of the auxiliary support rail mounted on a slider on the cubic framework. The symmetrical structure of upper and lower branches provide the characteristics of three-dimensional driving with six degree of freedom, coupling relationship not exist between the horizontal and vertical movement of sliders, it make the control simple, complex space surface manufacture can be realized, still high precision control and other issues solved, it will have a very broad application prospects. The equivalent diagram of mechanism as show in Fig.3 obtained by removing the non-rail portion and non-framework as show in Fig. 1, the kinematical pairs expressed by arabic numerals.
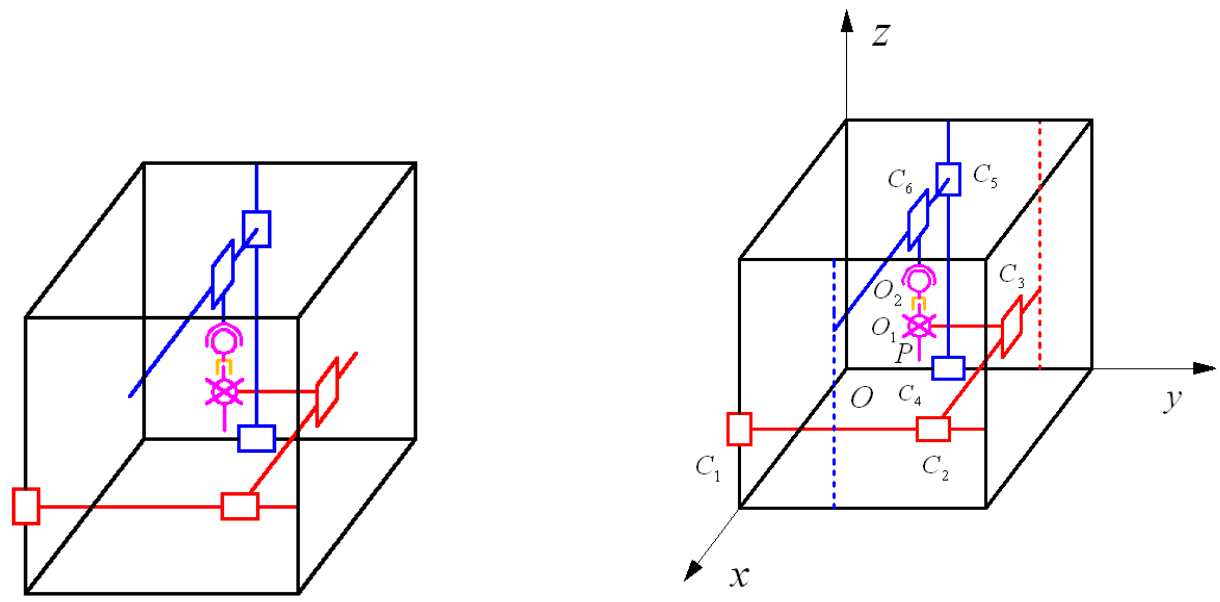

Fig. 1 Schematic diagram of hybrid cubic mechanism Fig. 2 Coordinates and the name of each part

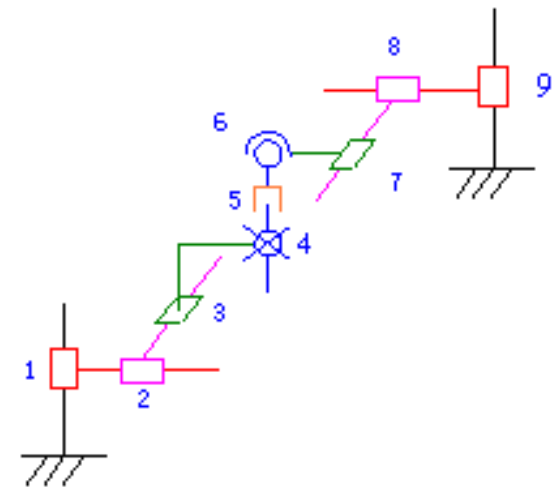

Fig. 3 Equivalent mechanism of PPP-S(P)[T]-PPP

\section{Analysis on Decoupling Capability of Input Parameters}

Input parameters are known to find output parameters defined as the forward solution of mechanism, position of input sliders given, position of the center of upper and lower platform can be obtained then, also the position $\left(X_{P}, Y_{P}, Z_{P}\right)$ and pose $\alpha, \beta, \gamma$ of output end found. The initial coordinates of slider $C_{i}$ on six moving guideway of cubic framework are $\left(x_{C_{i}}, y_{C_{i}}, z_{C_{i}}\right)(i=1,2,3,4,5,6)$ _respectively (here and below), the coordinate of output shaft end hooke joint and ball joint center are $\left(x_{O_{1}}, y_{O_{1}}, z_{O_{1}}\right)$ and $\left(x_{O_{2}}, y_{O_{2}}, z_{O_{2}}\right)$ respectively, the distance of $\left|O_{1} O_{2}\right|=t$ is variable, $\left|P O_{1}\right|=h$ is the distance of hooke joint to output shaft end point $\mathrm{P}$, coordinates of point $\mathrm{P}$ is $\left(x_{P}, y_{P}, z_{P}\right)$, the direction vector of output shaft expressed by $\left(k_{1}, k_{2}, k_{3}\right)$, 
the distance of hooke joint and slider $C_{3}$ is $\left|C_{3} O_{1}\right|=d y$ is fixed value, the distance of ball joint and slider $\mathrm{C}_{6}$ is $\left|\mathrm{C}_{6} \mathrm{O}_{2}\right|=d z$ is fixed value as well. Due to the special structure of mechanism, relationship exists between them as following.

$$
\begin{gathered}
\left\{\begin{array}{c}
x_{O_{1}}=x_{C_{3}} \\
y_{O_{1}}=y_{C_{2}}-d y \\
z_{O_{1}}=z_{C_{1}}
\end{array},\right. \\
\left\{\begin{array}{c}
x_{O_{2}}=x_{C_{6}} \\
y_{O_{2}}=y_{C_{4}} \\
z_{O_{2}}=z_{C_{5}}-d z,
\end{array}\right.
\end{gathered}
$$

Cosine angles of output shaft can be obtained from the forward solution of position of mechanism as follows,

$$
\left\{\begin{array}{l}
\alpha=\arccos \left(\left(x_{O_{2}}-x_{O_{1}}\right) / \sqrt{\left(x_{O_{2}}-x_{O_{1}}\right)^{2}+\left(y_{O_{2}}-y_{O_{1}}\right)^{2}+\left(z_{O_{2}}-z_{O_{1}}\right)^{2}}\right) \\
\beta=\arccos \left(\left(y_{O_{2}}-y_{O_{1}}\right) / \sqrt{\left(x_{O_{2}}-x_{O_{1}}\right)^{2}+\left(y_{O_{2}}-y_{O_{1}}\right)^{2}+\left(z_{O_{2}}-z_{O_{1}}\right)^{2}}\right) \\
\gamma=\arccos \left(\left(z_{O_{2}}-z_{O_{1}}\right) / \sqrt{\left(x_{O_{2}}-x_{O_{1}}\right)^{2}+\left(y_{O_{2}}-y_{O_{1}}\right)^{2}+\left(z_{O_{2}}-z_{O_{1}}\right)^{2}}\right)
\end{array}\right.
$$

If the position of center $\mathrm{O} 1$ of lower platform determined, that is $\left(x_{O_{1}}, y_{O_{1}}, z_{O_{1}}\right)$ assumed as constant, variable passive telescopic joint $(\mathrm{P})$ is assumed as a fixed state, $\left|O_{1} O_{2}\right|=t$ is constant, in order to change the pose of output shaft, we just need to change the input parameters of three sliders on upper platform independently, expression (3) can be written into

$$
\left\{\begin{array}{l}
\alpha=\arccos \left(\left(x_{C_{6}}-x_{O_{1}}\right) / t\right) \\
\beta=\arccos \left(\left(y_{C_{4}}-y_{O_{1}}\right) / t\right) \\
\gamma=\arccos \left(\left(z_{C_{5}}-z_{O_{1}}\right) / t\right)
\end{array} .\right.
$$

Due to the exists of variable passive telescopic joint $(\mathrm{P})$, when the center $\mathrm{O} 1$ move to a point in its working space, we just need to change the parameters of three sliders on lower platform independently, that is the control of $C_{i}(i=1,2,3)$. Due to the variable characteristics of $\left|O_{1} O_{2}\right|=t$, assuming the telescopic range is between 0 and a, when the center point $\mathrm{O}_{2}$ of upper platform move to another position, we just need to change the parameters of three sliders $C_{j}(j=4,5,6)$, independently.

Partial derivative of three output variables in formula (1) to three input variables on lower platform carried out, also partial derivative of three output variables in formula (2) to three input variables on upper platform carried out, still partial derivative of three output variables in formula (3) to three input variables on upper platform carried out, we will get $\frac{\partial x_{O_{1}}}{\partial x_{C_{3}}}=1, \frac{\partial y_{O_{1}}}{\partial y_{C_{2}}}=1$, $\frac{\partial z_{O_{1}}}{\partial z_{C_{1}}}=1, \frac{\partial x_{O_{2}}}{\partial x_{C_{6}}}=1, \frac{\partial y_{O_{2}}}{\partial y_{C_{4}}}=1, \frac{\partial z_{O_{2}}}{\partial z_{C_{5}}}=1$, and the rest results is 0 . Partial derivatives of cosine angle of output shaft to three input parameters on the upper layer platform as following,

$$
\frac{\partial \alpha}{\partial x_{C_{6}}}=-\frac{1}{\sqrt{1-\left(\left(x_{C_{6}}-x_{O_{1}}\right) / t\right)^{2}}} \cdot \frac{1}{t}
$$




$$
\begin{gathered}
\frac{\partial \beta}{\partial y_{C_{4}}}=-\frac{1}{\sqrt{1-\left(\left(y_{C_{4}}-y_{O_{1}}\right) / t\right)^{2}}} \cdot \frac{1}{t}, \\
\frac{\partial \gamma}{\partial z_{C_{5}}}=-\frac{1}{\sqrt{1-\left(\left(z_{C_{5}}-z_{O_{1}}\right) / t\right)^{2}}} \cdot \frac{1}{t},
\end{gathered}
$$

From the analysis and calculation results above we can see that the input parameters on upper or lower platform control the position and pose of output shaft independently, the position of point $\mathrm{O}_{1}$ decided by three sliders on lower platform, the position of point $\mathrm{O}_{2}$ decided by three sliders on upper platform, given the position of point $\mathrm{O}_{1}$, the pose of output shaft $\mathrm{O}_{1} \mathrm{O}_{2}$ decided by three sliders on upper platform, which control the cosine angle of output shaft.

\section{Simulation and Analysis of Example}

Relevant parameters given as following, $\quad a=1000 \mathrm{~mm}, t=100 \mathrm{~mm}, h=200 \mathrm{~mm}$, $d_{1}=200 \mathrm{~mm}, d_{2}=100 \mathrm{~mm}$, Assume the vertical height of output shaft end point P in the cubic mechanism is $300 \mathrm{~mm}$, With point $S:(500,500,300)$ as the center, planar circular motion around the vertical axis $\mathrm{O}_{Z}$ parallel to the horizontal surface with a radius of $200 \mathrm{~mm}$, direction cosine angle of output shaft $\mathrm{O}_{1} \mathrm{O}_{2}$ and axis $\mathrm{Oz}$ always $30^{\circ}$, The circumferential velocity of point $\mathrm{P}$ at initial position is $\omega_{P_{0}}=\frac{\pi}{18}(\mathrm{rad} / \mathrm{s})$, its angular acceleration is $\delta_{P}=0.1\left(\mathrm{rad} / \mathrm{s}^{2}\right)$, circular motion step of output end point assumes $5^{\circ}$, motion range is $\left[0,360^{\circ}\right]$. Response capacity of each input parameters to the position and pose of output shaft obtained by using simulation software and program analysis, as shown in Fig. 4 to Fig.6.

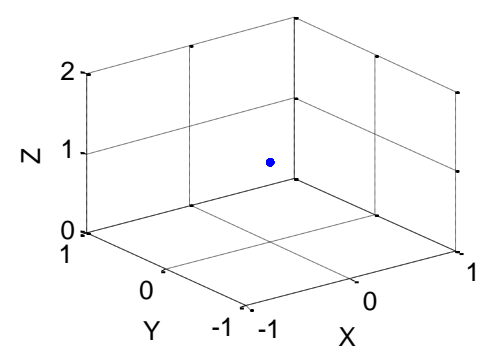

(a) dynamic response of point $\mathrm{C}_{1}$ for position of $\mathrm{O}_{1}$

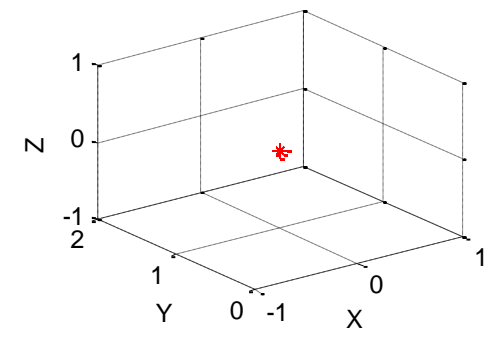

(b) dynamic response of point $\mathrm{C}_{2}$ to position of $\mathrm{O}_{1}$

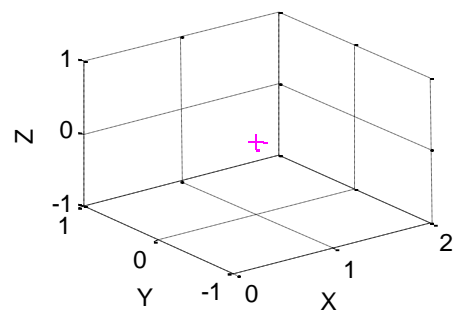

(c) dynamic response of point $\mathrm{C}_{3}$ to position of $\mathrm{O}_{1}$

Fig. 4 Dynamic response of each point on lower platform to position of $\mathrm{O}_{1}$

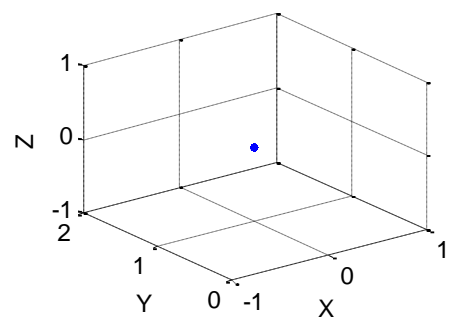

(a) dynamic response of point $\mathrm{C}_{4}$ to position of $\mathrm{O}_{2}$

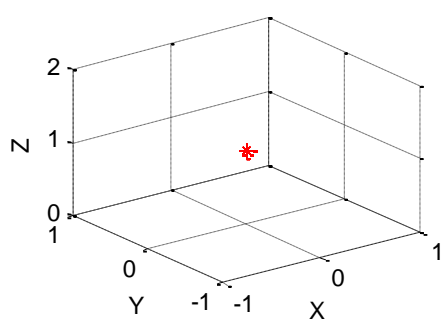

(b) dynamic response of point $\mathrm{C}_{5}$ to position of $\mathrm{O}_{2}$

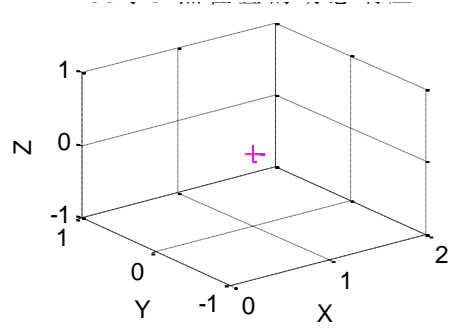

(c) dynamic response of point $\mathrm{C}_{6}$ to position of $\mathrm{O}_{2}$

Fig.5 Dynamic response of each point on upper platform to position of $\mathrm{O}_{2}$ 


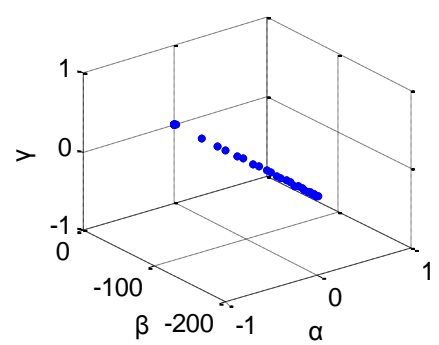

(a) dynamic response of point $\mathrm{C}_{4}$ to the pose of $\mathrm{O}_{1} \mathrm{O}_{2}$

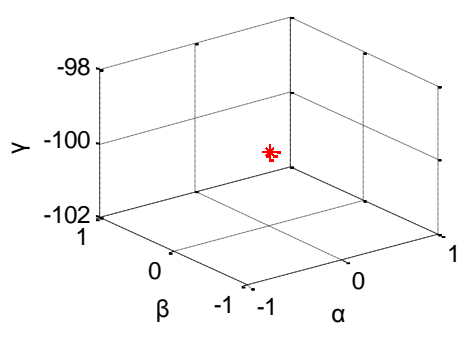

(b) dynamic response of point $\mathrm{C}_{5}$ to the pose of $\mathrm{O}_{1} \mathrm{O}_{2}$

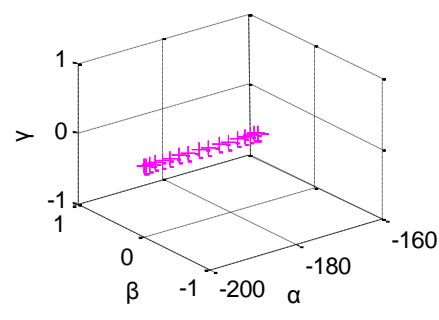

(c) dynamic response of point $\mathrm{C}_{6}$ to the pose of $\mathrm{O}_{1} \mathrm{O}_{2}$

Fig. 6 Dynamic response of each point on upper platform to the pose of $\mathrm{O}_{1} \mathrm{O}_{2}$

\section{Conclusion}

Following conclusion can be draw clearly by comparing the dynamic response value of the same input parameters to the position and pose of output shaft respectively or the sum of partial parameters.

(1) A decisive role exists in dynamic response of each input parameters on lower platform to the position of point $\mathrm{O}_{1}$ on output shaft, the input parameters of each motion slide corresponding to the position components of point $\mathrm{O}_{1}$ respectively.

(2) A decisive role exists in dynamic response of each input parameters on upper platform to the position of point $\mathrm{O}_{2}$ on output shaft, the input parameters of each motion slide corresponding to the position components of point $\mathrm{O}_{2}$ respectively.

(3) When the position of point $\mathrm{O}_{1}$ given, a decisive role exists in dynamic response of each input parameters on upper platform to the pose of output shaft $\mathrm{O}_{1} \mathrm{O}_{2}$, the input parameters of each motion slide corresponding to the pose components of output shaft $\mathrm{O}_{1} \mathrm{O}_{2}$ respectively.

(4) Six components of position change and pose change of output shaft realized by independent dynamic response of six input parameters correspondingly, perfect decoupling control achieved in position and pose.

\section{References}

[1] Z. Hao, W. Q. Yi. Analysis of parallel mechanism Stewart pose errorrr: submitted to Journal of Northeastern University( 2000).

[2] H. J. Jie, Z. J. Wei. Sensitivity analysis of the spindle platform on the structure parameter of the parallel robot: submitted to Journal of Jiaozuo Institute of Technology(2004).

[3] Y. S. Ping, L. D. Shi, L. S. Liang. Seven degree of freedom virtual axis on the design concept of parallel machine tool: submitted to Journal of North China Institute of Technology( 2004).

[4] Z. Wei, Y. J. Jun. Study on the parallel micromanipulator system: submitted to Journal of Beihang University(2001).

[5] R. J. Tie, S. H. Xu. Analysis of kinematics of three branch space robot with: submitted to Journal of Beihang University(2001). 